\title{
(In)soumise à la censure : l'activisme littéraire et politique de Suzanne Roussi-Césaire
}

Resisting Censorship: Suzanne Roussi-Césaire's Literary and Political Activism

Vanessa Lee

\section{(2) OpenEdition}

Journals

Édition électronique

URL : http://journals.openedition.org/transtexts/1518

DOI : $10.4000 /$ transtexts. 1518

ISSN : 2105-2549

Éditeur

Gregory B. Lee

\section{Référence électronique}

Vanessa Lee, «(In)soumise à la censure : l'activisme littéraire et politique de Suzanne RoussiCésaire », Transtext(e)s Transcultures 跨文本跨文化 [En ligne], 15 | 2020, mis en ligne le 14 décembre 2020, consulté le 27 janvier 2021. URL : http://journals.openedition.org/transtexts/1518 ; DOI : https:// doi.org/10.4000/transtexts. 1518

Ce document a été généré automatiquement le 26 janvier 2021.

(c) Tous droits réservés 


\title{
(In)soumise à la censure : l'activisme littéraire et politique de Suzanne Roussi-Césaire
}

\author{
Resisting Censorship: Suzanne Roussi-Césaire's Literary and Political Activism
}

Vanessa Lee

12018 marquait le dixième anniversaire de la mort d'Aimé Césaire, décédé le 17 avril 2008. Homme de lettres, théoricien, homme politique martiniquais, sa pensée et ses écrits ont à la fois marqué la littérature française et la pensée postcoloniale internationale. Sans doute est-il mieux connu pour avoir été l'un des chantres, avec le Sénégalais Léopold Sédar Senghor et le Guyanais Léon Gontran-Damas, de la Négritude, mouvement littéraire et théorique de l'identité et de l'art noirs. Des études récentes ont cherché à étudier la place des femmes qui inspirèrent, participèrent, ou écrivirent en parallèle à ce mouvement. L'une d'elles, Suzanne Césaire, née Suzanne Roussi, épouse d'Aimé de 1937 à 1963, fut l'une des pionnières de l'écriture féminine - et féministe antillaise.

2 Suzanne Roussi naît le 11 août 1915 en Martinique. Elle fait ses études supérieures à Toulouse puis à Paris, où elle rencontre Aimé Césaire, qu'elle épousera en 1937. Ils auront six enfants. Lors de leurs études à Paris, Suzanne et Aimé travaillent à la rédaction du journal L'Étudiant noir, expérience qui leur sera utile lorsque, de retour en Martinique pendant la Seconde Guerre Mondiale, ils fondent avec leurs amis enseignants René Ménil, Aristide Maugée, et Lucie Thésée, la revue Tropiques, dont quinze numéros seront publiés entre 1941 et $1945 .{ }^{1}$ Suzanne Césaire y contribua en publiant sept articles et fut aussi l'interlocutrice entre les services d'information du gouvernement pétainiste et le comité éditorial de Tropiques. Lorsque les censeurs interdirent la revue en 1943, ce fut Suzanne Césaire qui adressa aux autorités une lettre défendant la ligne éditoriale. Entre 1945 et 1963, la carrière politique d'Aimé Césaire embarque sa famille dans un va-et-vient entre la Martinique et la métropole. En 1952, Suzanne Césaire écrit une pièce de théâtre, Youma, ou l'aurore de la liberté, inspirée d'un roman de Lafcadio Hearn et jouée par les élèves du lycée Schœlcher à Fort-de-France. Il 
ne reste malheureusement aucune trace écrite de cette pièce, ni de ses autres adaptations dramatiques. ${ }^{2}$ En 1963, Suzanne se sépare de son mari et meurt trois ans plus tard, à l'âge de cinquante ans.

3 Même si son œuvre ne fut reconnue que plusieurs décennies après son décès, l'influence littéraire et théorique de Suzanne Césaire sur la littérature martiniquaise n'est pas négligeable. Ses textes parus dans Tropiques peuvent paraitre démodés et quelque peu simplistes, mais il faut se souvenir qu'elle écrivait dans un journal soumis à la censure et qu'il lui fallait, par conséquent, être discrète et allusive dans ses propos. Toutefois, de ses textes à l'apparence anodine et parfois d'un style ampoulé, surgissent des éclairs de lucidité, de pensée et d'inspiration dignes des plus belles envolées d'Aimé Césaire ou des images évocatrices d'Édouard Glissant. On peut citer, par exemple, l'expression «la poésie martiniquaise sera cannibale ou ne sera pas ", prônant la « consommation» de toutes les cultures du «brassage continu» desquelles l'identité caribéenne est le résultat, afin de créer une littérature martiniquaise à part entière. ${ }^{3}$ Empruntant une phrase célèbre du surréaliste André Breton, et qui sera reprise à son tour par René Ménil, ceci est un exemple de ses déclarations porteuses de toute une pensée littéraire et artistique. ${ }^{4}$

\section{Les écrivaines martiniquaises}

L'importance des écrivaines caribéennes de l'époque des Césaire ou les ayant précédés, commence à être progressivement reconnue. D'excellentes études sur la question sont parues au cours des dix ou vingt dernières années. ${ }^{5}$ Cela a donné lieu à l'établissement d'une généalogie de la littérature féminine caribéenne, "which at once matches and challenges 'l'héritage viril' of Antillean writing [qui est l'égale de, et remet en question, 'l'héritage viril' de la littérature antillaise]", et à la reconnaissance de plusieurs générations de femmes écrivains oubliées par la critique. ${ }^{6}$ Dans cette généalogie, on retrouve Paulette et Jeanne Nardal, vedettes des cercles intellectuels noirs à Paris dans les années 1920 et 1930, qui dans leurs salons littéraires accueillirent des membres du mouvement de la Harlem Renaissance, ainsi que les futurs fondateurs de la Négritude. Paulette et Jeanne écrivaient aussi, et l'un des textes de Paulette rédigé en 1932 témoigne avec lucidité de l'expérience des jeunes intellectuelles en France :

Les femmes de couleur vivant seules dans la métropole moins favorisées jusqu'à l'Exposition coloniale que leurs congénères masculins aux faciles succès, ont ressenti bien avant eux le besoin d'une solidarité raciale [...] : c'est ainsi qu'elles se sont éveillées à la conscience de race. [...] Après s'être docilement mises à l'école de leurs modèles blancs, peut-être sont-elles passées, comme leurs frères noirs américains, par une période de révolte. [...] Au cours de leur évolution, leur curiosité intellectuelle s'est tournée vers l'histoire de leur race et de leurs pays respectifs. C'est ainsi qu'elles ont été amenées à déplorer l'absence de cette intéressante matière dans les programmes d'enseignement appliqués aux Antilles [et] elles se sont mises à l'étude. ${ }^{7}$

Bien avant la Négritude, et en parallèle avec le mouvement de la Harlem Renaissance aux États-Unis, ces jeunes intellectuelles préparèrent le terrain pour toute une réflexion sur la colonisation, le racisme, ainsi que sur les questions de genre, qui allait se développer au cours des décennies suivantes. La « conscience de race " citée dans ce passage peut être rapprochée de l'idée de « double consciousness » de W.E.B. Dubois. Et 
l'idée d'étudier l'histoire de leur pays et de leurs ancêtres est également prônée par les mouvements tels la Harlem Renaissance ou de la Négritude.

Pourquoi Suzanne Césaire ne fut-elle pas reconnue plus tôt ? On peut comprendre que les figures telles que les Nardal, arrivèrent trop tôt, ou pour ce qui est des autres jeunes femmes dont il est question dans la citation ci-dessus, qu'elles n'étaient pas assez connues. Mais Suzanne était la femme d'un des plus grands écrivains du vingtième siècle. Et comme Suzanne Dracius le démontrait dans un article «In Search of Suzanne Césaire's Garden [À la recherche du jardin de Suzanne Césaire] ", la popularité de Sartre n'éclipsa pas pour autant celle de Simone de Beauvoir :

It did me no good to speak to [Aimé Césaire] about de Beauvoir, who had come, at the very same time, into that France of the twentieth century, with the help and support of Sartre, certainly with greater difficulty and much later than Sartre, perhaps, but even so, with success... From all evidence, what was good for Simone was not good for Suzanne. All women belong to the "second sex" but all don't write Le Deuxième sexe. Moreover, the problems, for a Martinican woman, a woman "of colour", a "daughter of the islands", are doubtless increased.

[Ça ne me servit à rien de parler à [Aimé Césaire] de de Beauvoir, qui était arrivée, à la même époque, dans cette France du vingtième siècle, avec l'aide et le soutien de Sartre, sans doute avec plus de difficulté et plus tard que Sartre, peut-être, mais tout de même, avec succès... De toute évidence, ce qui était bon pour Simone ne l'était pas pour autant pour Suzanne. Toutes les femmes appartiennent au "deuxième sexe», mais toutes n'écrivent pas Le Deuxième sexe. De plus, les problèmes, pour une Martiniquaise, une femme " de couleur », une «fille des îles ", sont sans doute exacerbés]. ${ }^{8}$

7 On peut spéculer quant à l'anonymat dans lequel fut plongée l'œuvre de Suzanne Roussi Césaire pendant des décennies. D’abord, Suzanne Césaire écrivit peu de textes. Il nous reste sept articles de Tropiques, ainsi qu'une méthode d'enseignement publiée dans les Cahiers d'Estienne. ${ }^{9}$ Malheureusement, il n'existe aucune trace des textes de ses pièces et adaptations théâtrales. De plus, elle est morte prématurément, cinq décennies avant Aimé. Toutefois, on peut argumenter qu'un certain nombre d'auteurs qui écrivirent peu ou moururent prématurément sont néanmoins reconnus par l'histoire littéraire. Suzanne Roussi Césaire était également mère de famille et enseignante, et c'est d'ailleurs pour se consacrer à ces occupations qu'elle ne poursuivit pas de carrière littéraire après Tropiques, selon sa famille. Elle avait aussi des problèmes de santé. Pourtant, à l'époque de Tropiques, elle était déjà enseignante et mère de famille nombreuse - elle avait quatre enfants - ce qui ne l'empêchait pas de participer activement à la vie de la revue. Mais il est tout à fait possible que le poids de ses responsabilités familiales et professionnelles, ajouté à sa santé défaillante, eut sans doute raison d'elle. Il est possible qu'elle se soit éclipsée, consciente du succès littéraire et du développement de la carrière politique de son mari.

8 Une autre explication, qui vaut aussi pour les femmes de la génération de Suzanne, ainsi que celle des Nardal, est donnée par Christine Dualé. Elle compare l'expérience des intellectuelles caribéennes du vingtième siècle à celle des Noires américaines du dix-neuvième :

... les Noires américaines ne purent jamais se ranger aux côtés des suffragettes blanches dans la lutte pour l'égalité, car en affirmant que seules les femmes étaient blanches et seuls les Noirs étaient hommes, seule l'expérience des femmes blanches fut prise en compte. [...] Les intellectuelles antillaises furent ensuite confrontées exactement aux mêmes écueils, car dans leur lutte contre l'impérialisme et le colonialisme, les intellectuels qui les entouraient ne firent finalement que 
reproduire les mêmes schémas des intellectuels dont ils s'inspiraient, et ils marginalisèrent ainsi le rôle de ces femmes très engagées. ${ }^{10}$ Roussi Césaire fonde avec son mari et leurs amis en 1941, à laquelle elle contribua par la
publication de sept écrits, et qu'elle représenta et défendit devant la censure. L'article
se conclura par une réflexion sur la pièce perdue, Youma aurore de la liberté.

\section{Tropiques}

11 En 1940, le Maréchal Pétain nomme l'Amiral Georges Robert comme représentant du régime de Vichy en Martinique. Il impose un régime de rigueur répressif. L'absence de commerce avec la France entraîne une pénurie de produits de première nécessité, qui sont remplacés par des productions locales. Maladies et mortalité s'accrurent pendant cette période, connue sous le nom «d'An tan Robè [au temps de Robert]». Dans ce "Vichy sous les tropiques », pour citer le titre de l'ouvrage d'Eric Jennings à ce sujet, le racisme institutionnalisé est de rigueur, les maires de couleur remplacés de force par des hommes politiques blancs locaux. ${ }^{11}$ La dissidence, comme on appelle la résistance aux Antilles, s'impose. Des milliers de jeunes gens fuient vers les possessions britanniques de Sainte-Lucie et de Dominique pour rejoindre les Forces Françaises Libres. En juillet 1943, l'Amiral Robert est contraint à fuir, et la Martinique rejoint la France libre.

12 Tropiques voit le jour en 1941, suite à l'initiative d'un groupe d'enseignants martiniquais, qui discernaient un «vide culturel » en Martinique, selon Aimé Césaire. Les textes portaient sur la faune et la flore martiniquaises, la littérature, l'histoire, la philosophie et l'anthropologie. Aimé Césaire, dans un entretien figurant dans l'édition de la revue complète parue en 1978, souligne la nécessité à l'époque de «faire une revue culturelle... Mais parce qu'on ne pouvait faire rien d'autre!» en raison de la censure du régime vichyste. ${ }^{12}$ Malgré le caractère "particulier» de leurs articles soumis à la censure, le désir des éditeurs de Tropiques était :

[d']appréhender dans le contexte martiniquais la réalité martiniquaise, la bien situer. Nous voulions que cette revue soit un instrument qui permette à la Martinique de se recentrer. Nous nous étions aperçus qu'il n'y avait rien dans ce domaine! Absolument rien! Alors, nous avons décidé d'étudier, systématiquement, la faune, la flore, etc. Même un régime très répressif ne peut pas vouloir nous empêcher de faire cela! Nous pensions qu'un tel programme serait de nature à aider les Martiniquais à acquérir une certaine conscience d'eux-mêmes. ${ }^{13}$

13 Le but de cette revue était de combler ce vide culturel dont parle Aimé Césaire, et de permettre aux Martiniquais de se (re)découvrir. La recherche de soi et de la formulation de l'identité caribéenne est un questionnement phare chez Suzanne Césaire. Ces articles paraissent éclectiques, mais derrière chacun se profile le désire d'arriver à ce but.

14 Le surréalisme fut une des manières d'élucider la question de comment concevoir et formuler cette identité martiniquaise. En 1941, débarquent en Martinique des artistes et écrivains à destination des États-Unis, fuyant l'Europe où la situation empire chaque 
jour. Parmi eux, Claude Lévi-Strauss, Wifredo Lam, et l'auteur du Manifeste du Surréalisme, André Breton. La rencontre entre les Césaire et Breton profita aux deux parties. Après le départ de Breton de la Martinique, Tropiques publie beaucoup plus d'articles sur le Surréalisme, et même plusieurs contributions de Breton lui-même. ${ }^{14}$

On peut comprendre pourquoi le Surréalisme fut apprécié par les écrivains caribéens. Le mouvement représentait une échappatoire aux codes artistiques et littéraires rigides qui jusqu'à présent avaient dicté leur formation intellectuelle ainsi que leur identité de colonisé. On peut citer le "marronnage » littéraire, inspiré du marronnage historique d'esclaves fugitifs qui s'enfuirent des plantations et créèrent des communautés autonomes et devenues mythiques. Le Marron a un statut singulier. Il est à la fois exclu de la société, marginal, ainsi que rebelle, autonome. La célèbre citation de Césaire " marronner, il faut marronner ", inspira plusieurs générations d'écrivains caribéens. Pour Édouard Glissant, penseur de la relation et du tout-monde, marronner revient à emprunter « les chemins de traverse de la pensée » et à éviter « les chemins bien dallés et bien goudronnés qui ont été tracés pour nous. ${ }^{15}$ Échapper, en d'autres termes, au carcan du style et de la pensée littéraire et philosophique traditionnelle.

D'un point de vue historique, pour les contributeurs de Tropiques, cette dimension rebelle du marronnage est une des facettes de la dissidence contre le régime vichyste. Alors qu'entre 4000 et 5000 Martiniquais et Guadeloupéens s'enfuirent vers les colonies britanniques aux Caraïbes pour rejoindre les Forces Françaises Libres, dans une sorte de marronnage physique, les écrivains de Tropiques se concentrèrent sur le marronnage de l'esprit. Ainsi, une nouvelle forme d'expression littéraire et artistique, exacerbée par la censure et l'autocensure, se profile dans les écrits de la revue. En effet, il ne faut pas oublier que les textes devaient être soumis aux censeurs du régime de l'Amiral Robert. Comme l'écrit René Ménil, coéditeur de la revue :

Le lecteur n'oubliera pas qu'à la censure circonstancielle d'État s'est naturellement surajoutée, dans l'écriture des écrivants de Tropiques, l'autocensure intérieure (freudienne) - censure préventive constante de la communauté et de l'individu (mœurs et préjugés, désirs et peurs) agissant à l'insu de l'écrivant et pesant sur l'écriture de tout le poids de l'inconscient. ${ }^{16}$

Toutefois, on peut discerner une évolution dans l'orientation thématique de Tropiques. Comme le dit Aimé Césaire «la situation devenant chaque jour plus intenable, nous avons dû prendre des positions de plus en plus politiques. Le problème culturel ne pouvait être posé en lui-même, ni être résolu par lui-même, il supposait un engagement politique. ${ }^{17}$ Cet enhardissement est discernable dans les textes que Suzanne Roussi Césaire publia dans la revue. On ressent l'impact, dans ses articles, d'évènements phares dans l'évolution de la revue et de la situation politique en Martinique.

18 La première contribution de Suzanne Roussi publiée dans la revue est intitulée "Léo Frobenius et le problème des civilisations ", et se concentre sur l'ethnologue Léo Frobenius et son analyse des différentes civilisations humaines. Ici, l'influence du Surréalisme n'est pas encore visible, quoique, selon Kara M. Rabbitt, l'article, qui fait alterner analyse et interprétations poétiques, nous offre " a glimpse of the essayist to come [un aperçu de l'essayiste à venir] ${ }^{18}{ }^{18}$ Ce qui ressort de cet article, toutefois, est la vision de l'Afrique comme approfondissement de la connaissance de l'Homme et « une vision de l'avenir " nécessaire pour interpréter "notre douloureuse époque », et bien entendu pour définir l'identité martiniquaise. ${ }^{19}$ Suzanne Césaire conclut en soulignant qu'il est « urgent d'oser se connaître soi-même, d'oser s'avouer ce qu'on est, d'oser se demander ce qu'on veut être $» .^{20}$ Cet article manifeste déjà un désir à travers l'analyse 
de l'œuvre de Frobenius qui » dépasse les raisonnements scolaires de ses prédécesseurs et de ses contemporains ", de chercher d'autres méthodes littéraires et scientifiques pour « se connaître soi-même ». ${ }^{21}$

19 L'article suivant de Suzanne Roussi est publié dans le deuxième numéro de Tropiques, qui paraît en juillet 1941 après le départ de Breton de la Martinique. L'article est une critique des théories du philosophe Alain - de son vrai nom Émile Chartier - sur l'art. À la fin de l'article, Césaire abandonne ce "guide", qui selon elle se perd dans les "chemins étroits et trop connus de la beauté traditionnelle», pour décrire une nouvelle approche pour "connaître l'inconnaissable» à travers l'art, approche notamment pratiquée par André Breton, qu'elle cite rapidement à la fin de l'article sans toutefois masquer son admiration pour le poète : «il convient de citer le nom d'André Breton, ses poèmes, son livre admirables : L'Amour fou $» .^{22}$

Le troisième article figure dans un volume de Tropiques auquel contribua Breton. Suzanne Césaire écrit un article dithyrambique, mi-philosophie, mi-poésie, sur André Breton, "André Breton, poète». Alors qu'elle critiquait les "chemins étroits» empruntés par Alain dans son précédent article, pour elle Breton mène ses lecteurs sur des routes « très claires où l'homme [...] voit clair, clair dans son passé qui est à la fois son avenir [...] connaissance totale. $»^{23}$ De plus, Césaire admire la vitalité et surtout la « joie » dont sont empreintes les œuvres de Breton, qui «dans l'horreur et l'angoisse même, [...] sait détecter les nappes souterraines de la joie. $»^{24}$

21 À partir du quatrième article, les propos de Suzanne Césaire sont plus virulents et axés sur la question de la culture et de l'identité martiniquaises, l'aboutissement des idées développées dans ses trois précédentes contributions. Dans "Malaise d'une poésie: John-Antoine Nau ", publié dans en janvier 1942, Suzanne Césaire critique l'exotisme des œuvres littéraires consacrées à la Martinique - connues aussi sous le nom de « littérature doudou » - et en particulier l'œuvre de John-Antoine Nau, qu'elle cite à plusieurs reprises dans le texte. Selon elle, Nau " passe à côté. Il regarde. Mais il n'a pas 'vu'. ${ }^{25}$ L'écrivain enchanté par «les 'charmes' de la vie créole» qui décrit " amoureusement nos paysages " passe à côté de l'expérience "nègre ", mais aussi du " 'merveilleux' du morne [colline dans la Caraïbe], son aura maléfique » et «sa dure promesse ${ }^{26}{ }^{26} \mathrm{~A}$ travers Nau, Césaire s'attaque aussi à toute une lignée d'écrivains utilisant l'exotisme dans leurs écrits sur les "colonies", et donne comme exemples Leconte de Lisle, José Maria de Heredia et Francis Jammes. Les dernières phrases de l'article sont parmi les plus citées et connues de l'œuvre de Suzanne Césaire :

Allons, la vraie poésie est ailleurs. Loin des rimes, des complaintes, des alizés, des

perroquets. Bambous, nous décrétons la mort de la littérature doudou. Et zut à

l'hibiscus, à la frangipane, aux bougainvilliers.

La poésie martiniquaise sera cannibale, ou ne sera pas. ${ }^{27}$

Avec cet article donc, Suzanne Césaire cherche surtout à encourager ses homologues martiniquais à rejeter la tendance de la littérature doudou pour faire place à une nouvelle littérature, qui sera à même de "regarder et voir» la réalité sociale, économique, culturelle et politique des gens ordinaires en Martinique. Et on constatera d'ailleurs, dans cet article et ceux qui suivent, que Suzanne Césaire ne cherche pas pour autant à tourner le dos aux paysages et à la flore martiniquais. Elle remet en question la vision coloniale et exotique des paysages, mais ne rejette pas pour autant leur « mystère ». Le " cannibalisme » littéraire que prône Suzanne Césaire cherche à créer 
une littérature inspirée de tous les aspects de l'expérience martiniquaise, qu'elle soit historique, coloniale, géographique ou culturelle.

On constate dans cet article que, par son impatience, son désir de se faire entendre, Suzanne Césaire commence à encourir dangereusement la censure. En effet, s'attaquer à la littérature doudou et à l'exotisme dans la littérature française revient à s'attaquer à une vision de la littérature « régionale » de la Martinique que les censeurs cherchaient à promouvoir en autorisant la publication de la revue Tropiques. Comme l'explique Anny Dominique Curtius, «[f]ifty years after Nau's travels to Martinique, doudouisme was vivid in Martinican literature as well as in the colonial mentality and the Vichyist propaganda tailored by Admiral Robert. [cinquante ans après le voyage de Nau en Martinique, le doudouisme était omniprésent dans la littérature martiniquaise ainsi que dans la mentalité coloniale et la propagande vichyste conçue par l'Admiral Robert.] $»^{28}$ De plus, ce terme de "cannibale», vision cauchemardesque de l'Autre sauvage, barbare, blasphématoire, qui toutefois est ici reprise, voire revendiquée, et utilisée de manière jubilatoire pour décrire cette nouvelle poésie, a de quoi inquiéter tout officier de gouvernement colonial qui se respecte.

Il n'est pas étonnant donc, qu'après la publication du cinquième numéro de Tropiques, la revue ait été censurée. Dans le cinquième article de Suzanne Césaire, paru en avril 1942, l'auteure élabore sa réflexion sur la culture et l'identité martiniquaises. Elle y critique l'assimilation des gens de couleur en Martinique, les empêchant de créer leur propre « expression de ce moi [collectif]», leur propre production culturelle..$^{29}$ Selon elle, «le Martiniquais a échoué parce que, méconnaissant sa nature profonde, il essaie de vivre d'une vie qui ne lui est pas propre. ${ }^{30}$ S'assimiler pour s'intégrer à la société coloniale est une chose, mais selon $\mathrm{S}$. Césaire, cette "réaction de défense contre une société oppressive» se serait internalisée, si bien que le Martiniquais "ne SAIT pas véritablement qu'il imite. Il ignore sa véritable nature, qui n'en existe pas moins. ${ }^{31} \mathrm{En}$ lisant cet article en conjonction avec "Misère d'une poésie », on peut déduire que l'imitation de la poésie de John-Antoine Nau et d'autres écrivains de l'exotisme est un type d'assimilation littéraire à laquelle se seraient adonnés les intellectuels martiniquais, et qu'il faudrait rejeter pour « nous connaître enfin nous-mêmes ». Et à la fin de l'article, Césaire explique comment former cette nouvelle culture et littérature martiniquaise :

Nous voici appelés à nous connaître enfin nous-mêmes, et voici devant nous les splendeurs et les espoirs. Le surréalisme nous a rendu une partie de nos chances. À nous de trouver les autres. À sa lumière. ${ }^{32}$

Bien qu'elle se soit référée au surréalisme et aux origines africaines des Martiniquais dans son texte, Suzanne Césaire est toutefois consciente que la culture martiniquaise est multiple, et ne devrait privilégier un héritage culturel et littéraire plus qu'un autre. Elle explique que cette recherche de l'identité doit bien entendu prendre en compte l'héritage africain, "que nous avons appris à connaître et à respecter ", mais qu'elle ne doit pas être un retour en arrière. ${ }^{33}$ Le futur de la société martiniquaise se trouve dans la célébration de son futur métissé, dans «la mobilisation de toutes les forces vives mêlées sur cette terre où la race est le résultat du brassage le plus continu $»{ }^{34}$ Le peuple martiniquais, ainsi que ceux de la majorité des îles et nations caribéennes, est la conséquence du "brassage» de plusieurs populations venues d'Afrique, d'Europe, d'Asie et du Moyen-Orient. ${ }^{35}$ Bien avant les créolistes, qui se penchèrent sur « la totalité kaléidoscopique » du métissage dans les Caraïbes, Suzanne Césaire s'efforçait de mettre 
en avant cette pluralité de la société martiniquaise, rejetant l'essentialisme d'un retour en arrière vers un héritage africain ancestral. ${ }^{36}$

Les articles de Suzanne Césaire parus après la déposition du régime vichyste en Martinique, qui mit par conséquent fin à la censure contre Tropiques, articulent plus clairement sa pensée autour des problèmes socio-économiques de la société martiniquaise, et de l'avenir de la littérature martiniquaise. Dans «Le surréalisme et nous ", Suzanne Césaire fait une mise au point sur le Surréalisme, réitérant sa capacité à « libérer l'homme en lui révélant son inconscient » et à aider « à libérer les peuples en éclairant les mythes aveugles qui les ont conduits jusqu'ici. $»^{37}$ L'article se divise en deux parties, l'une étant la critique du surréalisme en 1943, et la seconde se posant la question de l'avenir de la littérature martiniquaise. Un avenir dont la formulation s'est vue facilitée par la pratique du surréalisme, mais qui se projette au-delà. En effet, comme le suggère Mireille Rossello, le titre de l'article, "Le Surréalisme et nous", indique à la fois une association aussi bien qu'une distance avec le surréalisme, en opposant « 'us', that is Black Martinicans, apart from 'Surrealism' ['nous', c'est-à-dire les Martiniquais noirs, au 'Surréalisme'] ». La phrase à la fin de l'article "Surréalisme, corde raide de notre espoir ", » alludes to a fragile alliance between the two movements [fait allusion à l'alliance fragile entre les deux mouvements] ». De plus, trop d'emphase mise sur la relation entre Breton et les écrivains martiniquais reviendrait à une lecture encore trop « eurocentrique » de l'évolution de la littérature martiniquaise. ${ }^{38} \mathrm{Il}$ ne s'agit pas d'être une succursale du surréalisme, mais bien un mouvement à part qui s'en inspire mais ne lui doit pas tout.

Suzanne Roussi explique aussi que le surréalisme a permis aux intellectuels martiniquais de faire face à la censure :

Pas un moment au cours de ces dures années de la domination de Vichy, l'image de la liberté ne s'est ternie totalement ici et c'est au surréalisme que nous le devons. Nous sommes heureux d'avoir maintenu cette image aux yeux mêmes de ceux qui croyaient l'avoir rayée à tout jamais. Aveugles parce qu'ignorants, ils ne la voyaient pas rire insolente, agressive, à travers nos pages - lâches ensuite, quand ils comprirent, apeurés, honteux. ${ }^{39}$

On peut désormais s'attendre à des articles reflétant cette « image de la liberté » avec plus de brio et d'honnêteté après la déchéance des «ignorants » censeurs du régime Vichy, où Suzanne Césaire peut décrire clairement sa vision de l'avenir pour la littérature et la société martiniquaise.

Son dernier article, qui paraît dans le dernier numéro de Tropiques, est l'aboutissement des idées de Suzanne Roussi Césaire. Dans « Le grand camouflage ", elle procède à une description de la réalité caribéenne, cachée par le "grand camouflage » de l'exotisme et du colonialisme, critiqués dans "Misère d'une poésie ». Sa description couvre en détail des éléments paysagers de la géographie caribéenne, montrant le revers de "l'exotisme» de John-Antoine Nau, et comment la réalité socio-économique et culturelle peut être représentée dans un paysage local :

[...] le serf antillais vit misérablement, abjectement sur les terres de «l'usine » et la médiocrité de nos villes-bourgs est un spectacle à la nausée. En attendant les Antilles continuent d'être paradisiaques et ce doux bruit de palmes... ${ }^{40}$

31 Elle réitère qu'il ne faut pas oublier la réalité de la société antillaise malgré ces descriptions du « paradis » tropical de la Martinique, et conclut de façon mélancolique : « si mes Antilles sont si belles, c'est qu'alors le grand jeu de cache-cache a réussi, c'est 
qu'il fait certes trop beau, ce jour-là, pour y voir. $»^{41}$ Suzanne Roussi développe dans cet article le concept qui, plus tard, sera nommé «écopoétique ", pratiquée par plusieurs écrivains caribéens de la fin du vingtième et du vingt-et-unième siècles..$^{42}$

Ce qui est le plus frappant dans cet article est que Suzanne Césaire commence son récit par un rapprochement de la Martinique des autres îles caribéennes, dont Haïti et Porto Rico, et montre une volonté de mettre en avant l'expérience régionale et « archipélique » des Amériques. On retrouve ici une pensée coïncidant avec les théories que développera Édouard Glissant quelques décennies plus tard sur l'archipélisme, l'Antillanité, la relation, pour souligner les spécificités culturelles, historiques et politiques de la Caraïbe.

Les articles de Suzanne Césaire pour Tropiques montrent une évolution marquée par l'adaptation à la censure à ses débuts et la libération de la parole sur sa fin, une découverte et une adaptation du Surréalisme après la visite de Breton, et l'articulation d'une poétique martiniquaise. Malgré l'autocensure et la censure du régime, Suzanne Roussi Césaire est parvenue à articuler des théories sur la culture et la littérature martiniquaises qui marquèrent le monde intellectuel de son époque et anticipèrent les théories et mouvements de la fin du vingtième siècle.

\section{Face aux censeurs}

En plus de s'adapter à la censure, Suzanne Césaire se met directement dans la ligne de mire de cette dernière. Chargée de livrer les épreuves de Tropiques aux censeurs, ce fut aussi elle qui rédigea une lettre en réponse à l'interdiction de la revue en 1943. Le 10 mai 1943, le commandant de vaisseau Bayle adressa au comité éditorial de la revue un réquisitoire contre une "revue révolutionnaire, raciale, et sectaire $»^{43}$ Bayle y accuse les éditeurs de chercher à corrompre les esprits des jeunes dont ils ont la charge en tant que professeurs, et de faire appel à la révolte. Il les accuse d'ailleurs de manque de patriotisme pour une " patrie qui a été précisément pour eux une si bonne patrie ».

En bon agent du régime vichyste, il répond aux griefs des éditeurs en soulignant les valeurs progressistes, tolérantes et sociales de la France « du Maréchal ». Bayle prétend être en accord avec la ligne éditoriale de Tropiques qui consiste à écrire une revue sur la vie, les us et coutumes locaux, "un régionalisme non moins vigoureux et tout aussi souhaitable » que celui de Frédéric Mistral, auteur provençal. Bayle explique que le régime de l'Amiral Robert s'oppose à la « centralisation excessive, mal dont ont souffert toutes les provinces françaises » et qui a risqué «d'étouffer la personnalité, de lui substituer un être conventionnel et uniforme ».

Contre les préjugés de race critiqués par Tropiques, Bayle souligne que, depuis Victor Schœlcher, «la France s'est engagée dans une politique d'égalité raciale [...] plus profondément mise en avant que n'importe quel pays: de cette politique, vous constituez le vivant témoignage ». Comment en tant que fonctionnaires de l'État français, et ainsi parfaitement assimilés à la société française, Suzanne Césaire et ses homologues osent-ils s'attaquer à la patrie qui les a libérés du joug de l'esclavage et qui a promu l'égalité entre les races? Bayle souligne d'ailleurs que les problèmes que décrivent les contributeurs de Tropiques sont plutôt d'ordre "social que racial». Toutefois, il n'hésite pas à ajouter que toute révolte ou contradiction du régime mènerait au « libre déchaînement de tous les instincts, et de toutes les passions; c'est le 
retour à la barbarie pure et simple.» On peut lire dans ces propos une insulte raciste tacite, suggérant que contre la civilisation blanche du régime Pétainiste, les éditeurs de Tropiques encourageraient le retour à la « barbarie » des peuples colonisés.

La réponse, envoyée le 12 mai suivant, rédigée par Suzanne Césaire au nom du comité éditorial, détourne et ridiculise les arguments de Bayle :

À Monsieur le Lieutenant de Vaisseau Bayle

Monsieur,

Nous avons reçu votre réquisitoire contre Tropiques.

" Racistes ", "sectaires ", " révolutionnaires ", " ingrats et traîtres à la Patrie ",

"empoisonneurs d'âmes", aucune de ces épithètes ne nous répugne essentiellement.

«Empoisonneurs d'âmes » comme Racine, au dire des Messieurs de Port-Royal.

«Ingrats et traitres à notre si bonne patrie » comme Zola, au dire de la presse réactionnaire.

« Révolutionnaires » comme l'Hugo des « Châtiments ».

«Sectaires », passionnément comme Rimbaud et Lautréamont.

«Racistes», oui. Du racisme de Toussaint Louverture, de Claude Mac Kay et Langston Hughes - contre celui de Drumont et de Hitler.

Pour ce qui est du reste, n'attendez de nous ni plaidoyer, ni vaines récriminations ni discussion même.

Nous ne parlons pas le même langage.

Signé : Aimé Césaire, Suzanne Césaire, Georges Gratiant, Aristide Maugée, René Ménil, Lucie Thésée. ${ }^{44}$

38 À chaque époque ses censeurs. On note aussi dans cette lettre un désir de se placer parmi les auteurs phares de la littérature française, en insistant sur le fait qu'ils sont bien « Français », en réponse à l'insistance sur ce point de la lettre de Bayle (" retenons seulement le fait que vous êtes Français»). Et ce faisant, ils soutiennent leur appartenance à la France libre, celle d'avant la guerre, et pas du tout celle de Vichy, accusant Bayle lui-même de ne pas parler la « même langue » que Racine, Zola, Hugo et Rimbaud.

Suzanne Roussi Césaire était un membre fondateur et intégral de la revue Tropiques. Ses écrits, plus virulents sur la fin, marquent un des fondements de la pensée identitaire caribéenne. Malgré l'autocensure, les écrivains de Tropiques formèrent un nouveau langage identitaire et politique, et à travers leur combat, avec Suzanne comme fer de lance, ils montrèrent au régime vichyste qu'ils n'étaient pas une force anodine.

\section{Youma : défi à l'autocensure et à la censure historique?}

Suzanne Césaire écrivit d'autres œuvres, qui furent malheureusement perdues. Mais le contexte d'écriture, politique et historique d'une de ces œuvres perdues - la pièce de théâtre Youma: Aurore de la Liberté (1952) - mérite une attention toute particulière. Inspirée du roman de Lafcadio Hearn Youma, the story of a West-Indian Slave (1890), la pièce traite d'une révolte des esclaves après la déclaration de l'abolition de l'esclavage dans les colonies en $1848 .{ }^{45}$ Cette thématique est intéressante pour deux raisons. D'abord parce qu'elle traite de l'abolition acquise par le peuple lui-même, et non pas accordée par l'État. Jusqu'en 1998, la journée de commémoration de l'abolition de l'esclavage était celle de l'abolition mise en œuvre par les envoyés de la métropole aux colonies. ${ }^{46}$ Toutefois, après cette date, les anciennes colonies purent choisir leur propre 
date de commémoration. En Martinique, il s'agit du 22 mai, date suivant la révolte des esclaves qui poussa les autorités locales à déclarer l'abolition le 23 mai, avant l'annonce officielle prévue. Le choix du 22 mai, et non pas du 27 avril, date officielle célébrée en France jusqu'à ce que les départements d'outre-mer puissent choisir leur propre date de commémoration, et ni du 23 mai, autre date officielle, montre le désir de réclamer une date où les Martiniquais s'émancipèrent seuls. ${ }^{47}$

41 Ainsi, cette pièce de 1952 écrite par Suzanne Césaire marquait le rétablissement d'un fait historique ignoré, éclipsé par l'histoire nationale officielle, qui ne fut reconnu qu'en 1998, ce qui montre l'avance de Suzanne Césaire sur son temps. On peut parler de défi à la censure historique, auquel toute une lignée d'auteurs et d'artistes antillais se prêtèrent afin de résister à l'oubli de l'histoire locale, une histoire souvent marquée par la résistance contre le pouvoir colonial ou celui de la métropole. ${ }^{48}$

Ce choix de sujet pour la pièce est d'autant plus surprenant et téméraire qu'il intervient six ans après la départementalisation de la Martinique, défendue par le mari de Suzanne, Aimé Césaire, alors maire de Fort-de-France et député d'Outre-Mer à l'Assemblée. La départementalisation fit des quatre anciennes colonies françaises des départements à part entière, les rapprochant davantage du giron de la France, mais conférant à leurs habitants le statut de citoyen. La loi de départementalisation, promulguée le 19 mars 1946, se nomme même « la loi Césaire ».49 Depuis, les débats ne cessent autour de ce changement de statut, qui aurait pu aboutir en indépendance. C'est ainsi qu'avec cette pièce Suzanne Césaire ajouta son grain de sel au débat sur l'autodétermination politique et historique de la Martinique.

\section{Conclusion}

Même si l'œuvre de Suzanne Roussi-Césaire resta dans l'ombre jusqu'à récemment, son exemple inspira, directement ou indirectement, des générations successives d'auteurs. On peut citer Daniel Maximin, l'éditeur de l'ouvrage rassemblant les œuvres complètes de Suzanne Césaire, Le Grand camouflage, ainsi que l'auteur d'une pièce inspirée des écrits de l'écrivaine, Suzanne Césaire Fontaine Solaire, mise en scène en 2015 pour le centenaire de la naissance de Suzanne Roussi. On peut aussi citer les filles de Suzanne Césaire, Ina et Michèle, qui, comme plusieurs femmes de leur génération, eurent des carrières à succès. Lucide, tout en sachant que sa génération ne serait pas celle de "femmes qui choisissent» selon sa fille Ina, Suzanne Roussi Césaire s'assura que la prochaine le serait sans faute, en tant qu'enseignante, mère, et auteure. Il reste à espérer que les recherches sur Suzanne Roussi mèneront à la découverte d'autres auteures de sa génération. Et que d'autres œuvres de Suzanne Roussi Césaire sortiront de l'ombre dans les années à venir. 


\section{NOTES}

1. Sous-titrée "Journal de l'Association des Étudiants Martiniquais en France ", la revue fut publiée de 1935 à 1940. Le premier numéro est consultable sur le site Gallica de la Bibliothèque Nationale de France : https://gallica.bnf.fr/ark:/12148/bpt6k9785762s?rk=21459;2

René Ménil (1907-2004), philosophe et écrivain martiniquais, fut considéré comme l'un des penseurs phares de sa génération aux côtés de Césaire, Fanon et Glissant. Il contribua aussi à $L a$ Revue du monde noir/The Review of the Black World, fondée par les sœurs Nardal et publiée de 1931 à 1932, et fut l'un des fondateurs de Légitime Défense en 1932.

Aristide Maugée (1913-1967), militant et écrivain Martiniquais, contribua à L'Étudiant noir dans les années 1930 ; il fut maire de Gros-Morne en Martinique de 1959 à 1967.

Lucie Thésée (1896-1990 ?), poétesse, écrivaine et enseignante martiniquaise, publia ses poèmes dans quatre numéros de Tropiques. Il existe très peu d'informations à son sujet, mise à part sa contribution à Tropiques.

2. Suzanne Césaire aurait adapté pour ses élèves les œuvres d'Yva Léro (1912-2007).

3. Toutes les citations de Tropiques, sauf indication contraire, sont tirées de Le Grand camouflage : écrits de dissidence (1941-1945), Daniel Maximin (dir.), Paris, Éditions du Seuil, 2015.

Suzanne Césaire, «Misère d'une poésie », Tropiques, n, janvier 1942, in Le Grand camouflage, p. 66.

«Malaise d'une civilisation », Tropiques, n5, avril 1942, in Le Grand camouflage, p.75.

4. La phrase d'André Breton paraît dans L'Amour fou, Paris, Éditions Gallimard, 1937, p. 26 : « la beauté convulsive sera érotique-voilée, explosante-fixe, magique-circonstancielle ou ne sera pas ».

René Ménil conclut son article «Laissez passer la poésie » avec «La poésie martiniquaise sera cannibale. Ou ne sera pas. ", Tropiques $n^{\circ} 5$, avril 1942, in Le Grand camouflage, p. 109.

5. Voir Christine Dualé, «L'émergence de la pensée féminine et féministe antillaise : Des sœurs Nardal à Suzanne Roussi Césaire ", Africultures (26 novembre 2014), url: http:// www.africultures.com/php/?nav=article\&no=12564 [consulté le 16 décembre 2014].

Kara M. Rabbitt, « Suzanne Césaire's Significance for the Forging of a New Caribbean Literature », The French Review, 79.3 (février 2006), pp. 538-548.

T. Denean Sharpley-Whiting, Négritude Women, Minneapolis: University of Minnesota Press, 2002. Sam Haigh, «'Voix féminines/Voix féministes?': Women's Writing from the Francophone Caribbean », Francophone voices, Kamal Salhi (ed.), Exeter: Elm Bank, 1999, pp. 141- 156.

6. Haigh, “'Voix féminines/Voix féministes ?' », p. 154.

7. Paulette Nardal, "Éveil de la Conscience de Race/Awakening of Race Consciousness ", in La Revue du monde noir/ The Review of the Black World. 1931-1932 Collection complete, Préface de LouisThomas Achille, 6 vol., Paris, Jean-Michel Place, 1992, pp. 347-348.

8. Suzanne Dracius, «In Search of Suzanne Césaire's Garden », Research in African Literatures, 41.1 (printemps 2010), p. 156.

Toutefois, lors d'un entretien-rencontre de Suzanne Dracius avec l'auteure à la Closerie des Lilas, lieu phare de l'histoire littéraire et artistique française, où chaque table porte une plaque où est gravé le nom d'une personnalité artistique ou littéraire, nous nous aperçûmes que nous étions assises à la table de Sartre et de de Beauvoir. La plaque de de Beauvoir était presque illisible, alors qu'on discernait parfaitement le nom de Sartre sur la sienne. Suzanne Dracius remarqua qu'il s'agissait là d'une belle illustration de la place des femmes dans l'histoire littéraire, où des figures phares telles de Beauvoir se voient tout de même éclipsées par leurs homologues masculins.

9. Les Cahiers d'Estienne $n^{\circ} 15$, Paris, Collège Technique Estienne, 24 juin 1949. 
10. Christine Dualé, "L'émergence de la pensée féminine et féministe antillaise: Des sœurs Nardal à Suzanne Roussi Césaire», Africultures (26 novembre 2014), url: http:// www.africultures.com/php/?nav=article\&no=12564 [consulté le 16 décembre 2014].

11. Éric Jennings, Vichy sous les tropiques: la révolution nationale à Madagascar, en Guadeloupe, en Indochine : 1940-1944, Paris, Grasset, 2004.

12. «Entretien avec Aimé Césaire par Jacqueline Leiner », Tropiques 1941-1945, Paris, Jean-Michel Place, 1978, p. viii.

13. «Entretien avec Aimé Césaire par Jacqueline Leiner », p. ix.

14. Dans Tropiques $n^{\circ} 3$ (octobre 1941) : «Vigilance» et «La mort rose » du recueil Le Revolver à cheveux blancs, pp. 38-39, 40 ; « Pour Madame Suzanne Césaire », p. 41 ; et un extrait tiré des Vases communicants dans « Relire », p. 69.

Dans Tropiques $n^{\circ} 5$ (avril 1942): «La lanterne sourde: à Aimé Césaire, René Menil, Georges Gratiant », pp. 37-38.

Dans Tropiques n6-7 (février 1943) : «Petit intermède prophétique », Lettre d'Aristide Maugée, pp. 63-64.

Dans Tropiques n9 (mai 1944) : "Martinique, charmeuse de serpents ", " Un grand poète noir ", qui sera inclus comme préface de la version bilingue du Cahier d'un retour au pays natal (Bordas, 1947), pp. 119-124.

15. Les Hommes livres - Édouard Glissant, Jean-René Christiani (éE.) (Institut national de l'audiovisuel, 1993).

16. René Ménil, «Pour une lecture critique de Tropiques », Tropiques 1941-1945, Paris, Jean-Michel Place, 1978, p. xxvi.

17. « Entretien avec Aimé Césaire par Jacqueline Leiner », p. xxiv.

18. Kara M. Rabbitt, «Suzanne Césaire's Significance for the Forging of a New Caribbean Literature ", The French Review 79.3 (février 2006), pp. 538-548, p. 540.

19. Suzanne Césaire, «Leo Frobenius et le problème des civilisations », Tropiques ${ }^{\circ} 1$, avril 1941, in Le Grand camouflage, p. 39

20. Césaire, «Leo Frobenius et le problème des civilisations », p. 40.

21. Césaire, « Leo Frobenius et le problème des civilisations », pp. 39-40.

22. Suzanne Césaire, "Alain et l'esthétique », Tropiques n², juillet 1941, in Le Grand camouflage, p. 50.

Césaire, «Alain et l'esthétique », p. 51.

23. Suzanne Césaire, "André Breton, poète...", Tropiques $\mathrm{n}^{\circ} 3$, octobre 1941, in Le Grand camouflage, p. 56.

24. Césaire, «André Breton, poète... », p. 54.

25. Suzanne Césaire, «Misère d'une poésie : John-Antoine Nau », Tropiques n4, janvier 1942, in Le Grand camouflage, p. 64.

26. Césaire, « Misère d'une poésie : John-Antoine Nau », pp. 63, 65.

27. Césaire, « Misère d'une poésie : John-Antoine Nau », p. 66.

28. Anny Dominique Curtius, «Cannibalizing Doudouisme, Conceptualizing the Morne: Suzanne Césaire's Caribbean Ecopoetics », The South Atlantic Quarterly 115.3 (juillet 2016), p. 518.

29. Suzanne Césaire, «Malaise d'une civilisation», Tropiques $n^{\circ} 5$, avril 1942, in Le Grand camouflage, p. 67.

30. Césaire, « Malaise d'une civilisation », p. 72.

31. Césaire, "Malaise d'une civilisation ", pp. 73, 74.

32. Césaire, « Malaise d'une civilisation », p. 75.

33. Césaire, « Malaise d'une civilisation », p. 75.

34. Césaire, «Malaise d'une civilisation », p. 75.

35. Après l'abolition de l'esclavage au dix-neuvième siècle, les gouvernements coloniaux firent venir de la main-d'œuvre d'Inde et de Chine dans les colonies de la Caraïbe, mais aussi en Guyane 
et à la Réunion. Au tournant du vingtième siècle, des immigrés venus de l'actuelle région du Liban et de la Syrie, vinrent s'installer en Martinique et s'ajoutèrent au «brassage » de la population.

36. Jean Bernabé, Patrick Chamoiseau, et Raphaël Confiant, Éloge de la créolité, Paris, Gallimard, 1993, p. 27.

37. Suzanne Césaire, « 1943 : Le surréalisme et nous », Tropiques n8-9, octobre 1943, in Le Grand camouflage, p. 81.

38. "Introduction by Mireille Rosello ", in Aimé Césaire, Notebook of a Return to My Native Land / Cahier d'un retour au pays natal, Traduction de Mireille Rosello et Annie Pritchard, Newcastle upon Tyne : Bloodaxe Books, 1995, p. 34.

39. Césaire, « 1943 : Le surréalisme et nous », p. 82.

40. Césaire, «Le grand camouflage », Tropiques $n^{\circ} 13-14,1945$, in Le Grand camouflage, p. 89.

41. Césaire, « Le grand camouflage », p. 94.

42. Voir Elizabeth DeLoughrey, Renée K. Gosson, et George B. Handley, Caribbean Literature and the Environment: Between Nature and Culture, Charlottesville: University of Virginia Press, 2005.

Daniel Maximin, Les Fruits du cyclone : Une géopoétique de la Caräibe, Paris, Seuil, 2006.

43. La lettre est reproduite dans Daniel Maximin, "Suzanne Césaire, Fontaine Solaire ", in Le Grand camouflage, pp. 12-13. Les citations sont tirées de cette reproduction.

44. Lettre reproduite dans « Suzanne Césaire, Fontaine Solaire », in Le Grand camouflage, pp. 13-14. 45. Il s'agit de la seconde abolition de l'esclavage dans les colonies françaises. L'esclavage fut d'abord aboli après la Révolution en 1794, puis rétabli par Napoléon Bonaparte en 1802.

46. Voir Chris Bongie, 'A Street Named Bissette : Assimilating the Cent-cinquantenaire of the Abolition of Slavery in Martinique (1848-1998)' in Friends and Enemies: The Scribal Politics of Post/ Colonial Literature (Liverpool : Liverpool University Press, 2008).

47. Pour une étude plus avancée des inspirations historiques et littéraires de Youma, voir Kara M. Rabbitt, « History into Story: Suzanne Césaire, Lafcadio Hearn, and Representations of the 1848 Martinique Slave Revolts ", Anthurium: A Caribbean Studies Journal 12.2, article 3 (2015), url: https://anthurium.miami.edu/articles/abstract/10.33596/anth.291/ [consulté le $1^{\text {er }}$ avril 2018].

48. Pour en savoir plus, voir Yarimar Bonilla, Non-sovereign Futures: Caribbean Politics in the Wake of Disenchantment (Chicago: University of Chicago Press, 2015).

Xavier-Marie Bonnot et François-Xavier Guillerm, Le Sang des nègres, Paris, Éditions Galaade, 2015. Louise Hardwick, « Depicting social dispossession in Guadeloupe: Nèg Maron, Lettre ouverte à la jeunesse and the general strike of 2009 », Forum of Modern Language Studies 48 (2012), pp. 288-305. 49. La loi Lamine Guèye du 7 mai 1946 conféra aux habitants des départements d'outre-mer le statut de citoyen.

\section{RÉSUMÉS}

Cet article se penche sur les écrits et l'activisme de Suzanne Césaire pendant la période de Tropiques et sur sa relation avec la censure politique, littéraire et sociale. Aimé Césaire fut l'un des écrivains majeurs du vingtième siècle. Écrivain, homme politique, théoricien, il fut l'un des chantres de la Négritude, mouvement littéraire et théorique de l'identité et l'art noirs. Suzanne Césaire née Roussi, épouse d'Aimé de 1937 à 1963, décédée prématurément à l'âge de 50 ans, fut l'une des pionnières de l'écriture féminine (et féministe) caribéenne. Avec son mari et d'autres 
intellectuels martiniquais, elle fonda Tropiques, revue culturelle publiée de 1941 et 1945 dans la Martinique de Vichy. Elle y contribua en publiant sept articles sur la psychanalyse, le Surréalisme, l'exotisme et l'identité antillaise. Elle fut aussi l'interlocutrice entre les services d'information du gouvernement pétainiste et le comité éditorial de Tropiques.

This article focusses on Suzanne Césaire's writings and activism during the Tropiques years, and her dealings with political, literary and social censorship. Aimé Césaire was one of the major literary figures of the twentieth century. A writer, politician and theorist, he was one of the founders of the literary and theoretical movement celebrating black identity and culture called Negritude. Suzanne Césaire, born Roussi, Aimé Césaire's wife from 1937 to 1963, was one of the pioneers of Caribbean women's and feminist literature. Along with her husband and a group of Martinican intellectuals, she co-founded the journal Tropiques, published between 1941 and 1945, when Martinique was under the control of the Vichy government. She contributed seven articles to the journal, on psychoanalysis, Surrealism, exoticism, and Caribbean identity. She also represented the Tropiques editorial board in its transactions with Vichy government censors.

\section{AUTEUR}

\section{VANESSA LEE}

Vanessa Lee est chercheuse et écrivaine de théâtre. Elle s'intéresse aux littératures, théâtres et cinéma postcoloniaux, aux études de genre, ainsi qu'aux théâtres européens, asiatiques, et caribéens. Ses articles figurant dans Global Media Journal ; The Journal of Romance Studies, Transtextes Transcultures, Bulletin of Francophone Postcolonial Studieset le Dangerous Women Project de l'Université d'Édimbourg. Site web: www.vanessalee.org

Vanessa Lee is an academic and playwright whose research interests include postcolonial theatre and film, gender studies, and European, Asian, and Caribbean theatres. Her articles have appeared in the Global Media Journal ; Journal of Romance Studies ; Transtext(e)s Transcultures; Bulletin of Francophone Postcolonial Studies and the University of Edinburgh's Dangerous Women Project. Personal webpage : www.vanessalee.org 